\title{
An experiment teaching method based on the Optisystem simulation platform
}

Jihua Zhu, Xuanlu Xiao, Yuan Luo

Jihua Zhu, Xuanlu Xiao, Yuan Luo, "An experiment teaching method based on the Optisystem simulation platform," Proc. SPIE 10452, 14th Conference on Education and Training in Optics and Photonics: ETOP 2017, 104525V (16 August 2017); doi: 10.1117/12.2269886

SPIE Event: 14th Conference on Education and Training in Optics and Photonics, ETOP 2017, 2017, Hangzhou, China 


\title{
An experiment teaching method based on the Optisystem simulation platform
}

\author{
ZHU Jihua*, XIAO Xuanlu, LUO Yuan \\ School of Optoelectronic Engineering, Chongqing University of Posts and telecommunications, \\ Chongqing, China, 400065
}

\begin{abstract}
The experiment teaching of optical communication system is difficult to achieve because of expensive equipment. The Optisystem is optical communication system design software, being able to provide such a simulation platform. According to the characteristic of the OptiSystem, an approach of experiment teaching is put forward in this paper. It includes three gradual levels, the basics, the deeper looks and the practices. Firstly, the basics introduce a brief overview of the technology, then the deeper looks include demoes and example analyses, lastly the practices are going on through the team seminars and comments. A variety of teaching forms are implemented in class. The fact proves that this method can not only make up the laboratory but also motivate the students' learning interest and improve their practical abilities, cooperation abilities and creative spirits. On the whole, it greatly raises the teaching effect.
\end{abstract}

Keywords: Experiment Teaching; OptiSystem; Simulation Platform; Demoes; Team Seminars

\section{INTRODUCTION}

The experimental teaching of optical communication system is difficult to achieve because of expensive equipment. So an approach of the experimental teaching based on the OptiSystem simulation platform is put forward in this paper.

OptiSystem is an innovative optical communication system simulation package that designs, tests, and optimizes virtually any type of optical link in the physical layer of a broad spectrum of optical networks, from analog video broadcasting systems to intercontinental backbones. OptiSystem is a stand-alone product that does not rely on other simulation frameworks. It is a system level simulator based on the realistic modeling of fiber-optic communication systems. It possesses a powerful new simulation environment and a truly hierarchical definition of components and systems. Its capabilities can be extended easily with the addition of user components, and can be seamlessly interfaced to a wide range of tools. ${ }^{[1]}$ What's more, OptiSystem has hierarchical definition system, intuitive graphical user interface and clearly classified device library, students can use this software to get started quickly and accomplish photonic device design, optical communication system construction and optical network design and analysis. In short, OptiSystem is suitable for assisting teaching and as a tool for students' daily practice. ${ }^{[2,3]}$

Firstly, the teacher introduces the software briefly based on the characteristic of the OptiSystem software, explain related knowledge points through the classic case and guide students to get familiar with the operation of the software. Then Students can go through class discussions to complete the example design in the form of groups. Lastly, teachers are going to assess and score according to the completion of personal report, team report, teachers' comments and peers review. This method can achieve student-oriented, so that it benefits to the improvement of student's self-learning ability and student's subjective initiative shall be fully developed.

\section{TEACHING METHOD}

Teaching method is one of the important ways to spread knowledge and to achieve the teaching objectives, which plays a big role in helping teachers and students to achieve the teaching tasks in the teaching process. ${ }^{[4]}$ The traditional teaching that always uses the method "one chalk, one blackboard", lacking in innovation and efficiency, seems to be unable to

*zhujh@cupt.edu.cn; phone 8613983606641

14th Conference on Education and Training in Optics and Photonics: ETOP 2017, edited by Xu Liu,

Xi-Cheng Zhang, Proc. of SPIE Vol. 10452, 104525V · C 2017 ICO, IEEE, OSA, SPIE

CCC code: $0277-786 X / 17 / \$ 18 \cdot$ doi: $10.1117 / 12.2269886$

Proc. of SPIE Vol. 10452 104525V-1 
meet the learning requirements and interests of quite a few students. As a consequence, an increasing number of teachers are trying to take the experimental teaching approach. Moreover, since a part of course emphasize the continuity and integrity instead of the systematic and scientific teaching methods, students still in a passive state during the teaching activities. ${ }^{[5]}$

Next, this paper will propose the improvement in three aspects: the teaching content, the teaching mode and the assessment method.

\subsection{The teaching content}

For example, referring to the Optical Fiber Communication Technology, the teaching content is divided into five topics: Optical fiber technology, Optical amplification technology, Dispersion management technology, Wavelength division multiplexing technology and Wavelength conversion technology. First of all, introduce the core components of the optical fiber and its attenuation, dispersion, nonlinear effects and other transmission characteristics. Then based on these characteristics introduce the corresponding technologies and solve some problems. According to the basic knowledge, the classic case and simulation, student teams should complete a simulation design based on the OptiSystem software. At last, the process and results should be show in class.

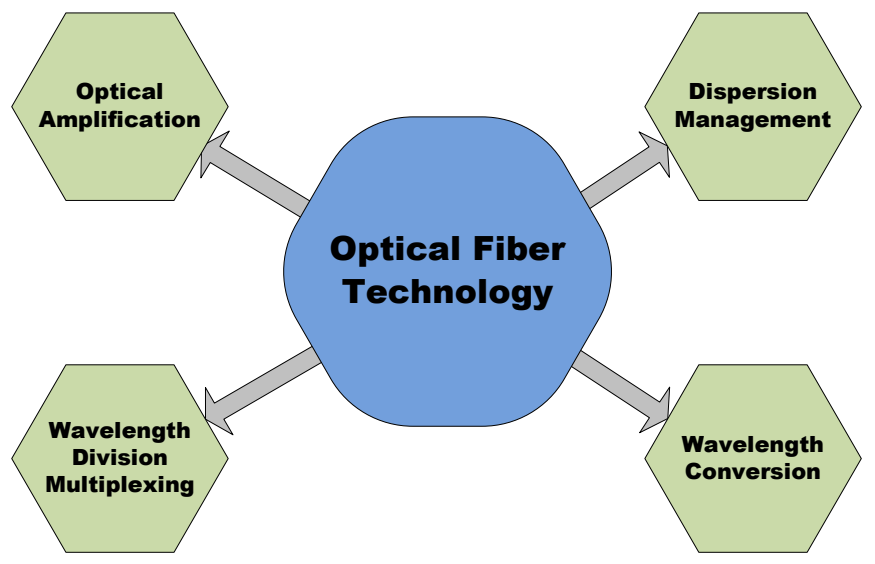

Figure 1. The teaching content

\subsection{The teaching mode}

At present, due to the improvement of talent requirements, students' self-learning and subjective initiative arouse the attention of colleges and universities. The experimental teaching method in this paper will make student-oriented, the teacher guide from the side. Teachers should encourage students to communicate each other by talk, information communication and feedback to implement teaching plan and accomplish teaching target.

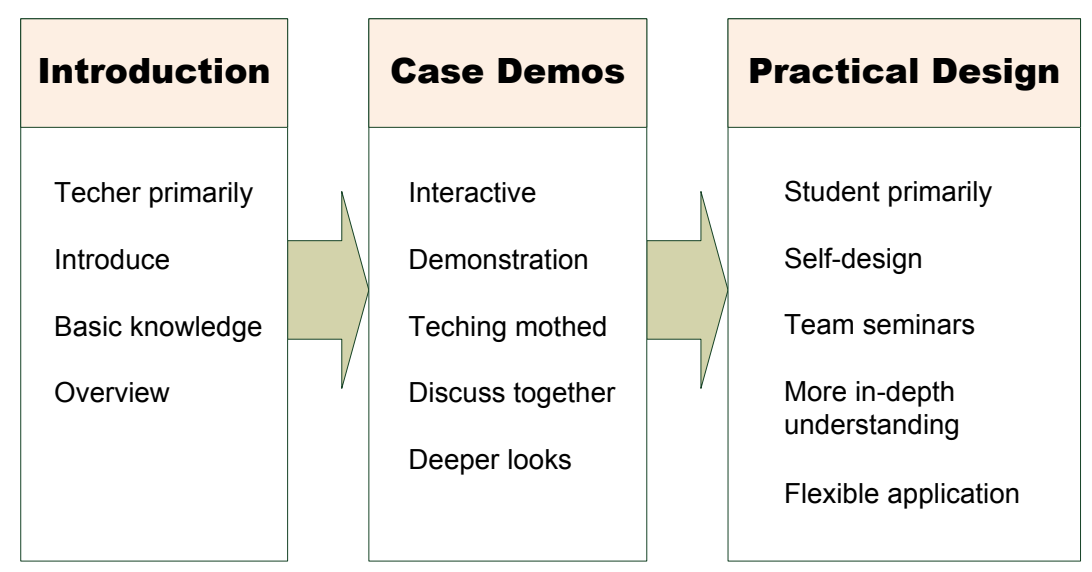

Figure 2. The teaching mode 
Firstly, the teacher introduces the OptiSystem software briefly based on the characteristic of the OptiSystem software, explain related knowledge points through the case and guide students to get familiar with the operation of the software. Then, set up the learning groups, which including excellent and poor students. The group organizes team tasks and assigns extracurricular time around the course topics, make all members search for information and finish the corresponding task with the plan, and finally finish the example design. With the process of gaining knowledge and expand learning area and the operation of software, students would not only understand and master the basic knowledge of the theory, but combine the knowledge and the practical application flexibly as well.

So that it benefits to the improvement of student's self-learning ability and student's subjective initiative shall be fully developed. All in all, student's expressing and communication skills are gotten exercise, the ability of learning and teamwork also improved in the "mutual aid" team atmosphere.

\subsection{The assessment method}

According to the requirement of the practice operation, we consider the method, containing the teachers' comments and peers review, which would promote students to find problems, make up for deficiencies and learn from each other. ${ }^{[6]}$ In addition, establish the competition within the group and between groups. The former is that group members scoring each other base on the team contribution, and the latter adopt a form of the oral examination, which each group will design courseware and show to all students in the last class. After display, students will answer questions about the key or the difficulty of the content, and teachers guide and comment due to the innovation, the integrity of the report and the performance of the display and so on. At last, all students are supposed to vote for selecting excellent team, to determine the final score.

This method not only allows each student to participate in the course, improve the efficiency of the course, but also achieves the purpose of training students' independent thinking ability and teamwork ability. In addition, it is conducive to the students' greater understanding of relevant knowledge, which stimulates the interest and initiative, and thus improves students' comprehensive application ability.

\section{EDFA GAIN CHARACTERISTICS SIMULATION}

Optical amplification technology is the key technology in optical fiber communication, which providing optical signal gain to compensate the transmission attenuation of optical signals in the fiber and increase the without-relay transmission distance of the system. An optical amplifier is a device that amplifies an optical signal directly, without the need to first convert it to an electrical signal, which is the core device for optical amplification in the optical transmission system. The appearance of Erbium-doped fiber amplifier (EDFA) is regarded as the important milestone in the history of fiber communication development, not only because of the high gain, low noise and good stability, but also can effectively support the increase in wavelength division multiplexing optical communication capacity. ${ }^{[7]}$ As the device that would be widely exposed in the future work, EDFA being an important teaching content in the part of optical amplification technology.

Rounding analysis of the EDFA gain characteristic, the following example will introduce the optical amplification technology based on the OptiSystem simulation platform.

In the first stage, basic knowledge of optical amplification technology is summarized briefly, the content containing the characteristics of optical amplification technology, some classic devise such as EDFA, Fiber Raman Amplifier (FRA), Semiconductor Optical Amplifier (SOA) and so on, the structure of the EDFA, the mechanism of amplification, etc. which mainly introduced by teachers in this stage, students will have a more comprehensive understanding about optical amplification technology.

In the second stage, the structure and properties of EDFA are discussed, and the gain characteristics, the noise characteristics and pump requirements of erbium - doped fiber amplifier are analyzed. The cases are shown in this stage, including case analysis and simulation by the OptiSystem simulation platform, deep in the relevant knowledge point, so that students should have a deeper understanding of the optical amplification technology especially the EDFA gain characteristics, and should know how to work the EDFA.

As showed in Fig.3. The teacher demonstrates how to select the signal light source, the pumping light source, the multiplexer, the erbium-doped fiber and other corresponding devices to build the erbium-doped fiber amplifier in the Optisystem. Before the analysis of gain characteristic, the parameter of device should be set according to the mechanism 
of the optical amplification of EFDA, such as the wavelength and power of signal light source and pump light source, the length of erbium-doped fiber, etc. And students should understand the basis for parameter settings.

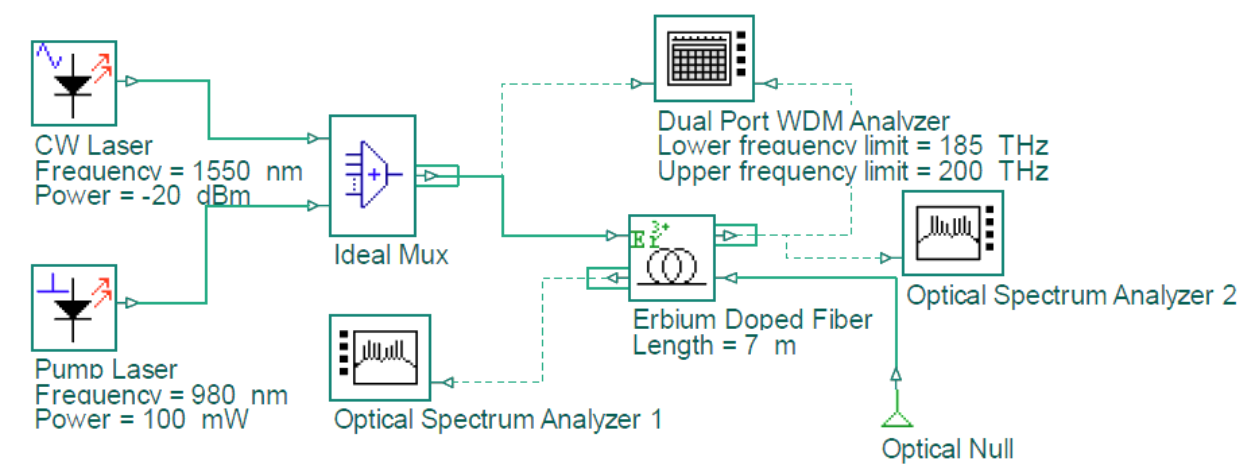

Figure 3. The structure of Erbium-doped Fiber Amplifier

In the measurement of gain characteristics, it is necessary to measure the power change of the optical signal before and after the erbium-doped fiber amplifier, and then calculate the gain value of the EDFA, which refers to how to measure the power of the optical signal, and what instrument to measure. Different measuring instruments have their own advantages, for example, the optical power meter can directly get the power data of the measurement point, the spectral analyzer can be more intuitive to obtain the measurement point of the optical power distribution, dual port wavelength division multiplexing analyzer can measure the power change between two measure port, which requires us to set the corresponding measurement instruments and get the appropriate measurement data.

In the analysis of gain characteristics, it is necessary to understand the gain to be effected by which factors, the relationship between them, these problems need exploring and studying. The gain of EDFA has a very complex relationship with the gain of EDFA, the pump power of the amplifier, the parameters of the doped fiber, and the input optical signal. Nothing can come into be at once. In analysis process, teachers would guide students to learn from shallow to deep, therefore students should consider these aspects in case design, and change a parameter under the same conditions, observe the amplifier gain trends, to understand the relationship between the amplifier gain and a single parameter changes. Then the following part will introduce Sweep mode which can achieve the mentioned condition in the Optisystem platform.

Sweep mode is a very useful function in the Optisystem platform, similar to enumeration method. For example, we need to understand the P-I characteristics of the laser in the general experiment, finding the relationship of the output power of the laser (P) and drive current (I). Sweep mode achieve the function by adjusting the current in accordance with certain laws within a certain range, and then under different I to measure P respectively in the Optisystem platform, which not only can manually set the corresponding data points, but also use the Spread tool in accordance with the linear, exponential, logarithmic, Gaussian, random and other functions to set the form, or even self-scripting custom settings.

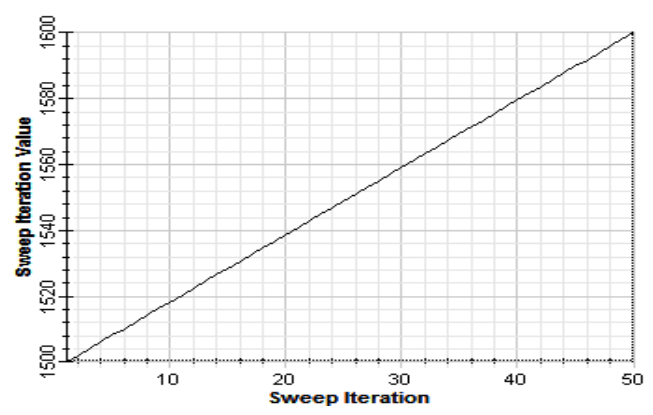

Figure 4. Sweep mode wavelength setting

For example, when we analyze the gain characteristic, we discuss the gains of the different wavelength signal light generated by EDFA. As shown in Fig.4, we take 50 points from the wavelength of $1500 \mathrm{~nm}$ to $1600 \mathrm{~nm}$ with the linear function of Sweep mode. In the signal light power of $-20 \mathrm{dBm}$, pump light power of $100 \mathrm{~mW}$, pump light wavelength of 
980nm, EDF length of $7 \mathrm{~m}$ and other conditions remain unchanged, we can get 50 sets of gain values after 50 different wavelengths of signal light through EDFA from the dual-port wavelength division multiplexing analyzer, and we can draw the EDFA gain $(G)$ with the signal light wavelength $(\lambda)$ change curve through the Report window in the Optisystem platform, which intuitively show the relationship between $G$ and $\lambda$, it is the EDFA Gain Spectrum Curve, as shown in Fig.5. From the gain spectrum curve, we can see when the wavelength is equal to $1520 \mathrm{~nm}$ the value of the amplifier gain can reach about $20 \mathrm{~dB}$. At the point A of $1531.4 \mathrm{~nm}$, the gain reaches the maximum value of $36.1 \mathrm{~dB}$. Between the point $\mathrm{B}$ and $\mathrm{C}$, the wavelength is from $1538 \mathrm{~nm}$ to $1558 \mathrm{~nm}$, the gain is stable at about $33 \mathrm{~dB}$ with good gain flatness, which is very suitable for multi-channel optical signal amplification in the wavelength division multiplexing system. Similarly, we can also draw the output power curve of signal light and noise figure curve, as shown in Fig.6.

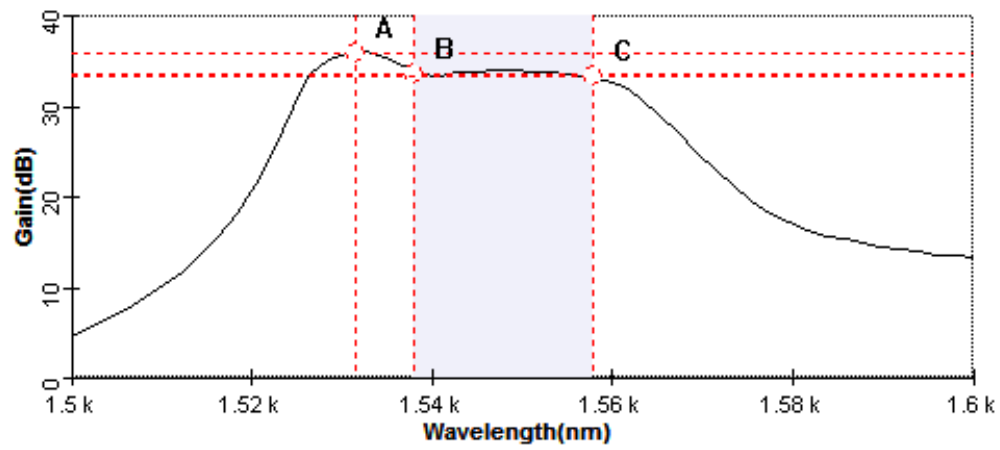

Figure 5. Gain spectrum curve $(\mathrm{G}-\lambda)$

In the third stage, students are supposed to learn by analogy, in-depth study the influence of the EDFA gain through changing the input signal optical power, pump optical power, the length of doped fiber parameters. And further expand the content, require students accomplish simple example design and analysis independently. In this stage the aim is that students can operate Optisystem software and complete the practical design by searching for the relevant references, group discussions, working together, and display and demonstrate in the last class. So students can have a more in-depth understanding of optical amplification technology, in particular EDFA gain characteristics. Students should complete the whole process through their own. In this process, teachers do encourage students to use a variety of ways to try, so that students can experience the optical fiber communication design process, find and analyze the problems from the error, then solve the problems. Finally students will deeply understand the optical fiber communication systems and related technologies.

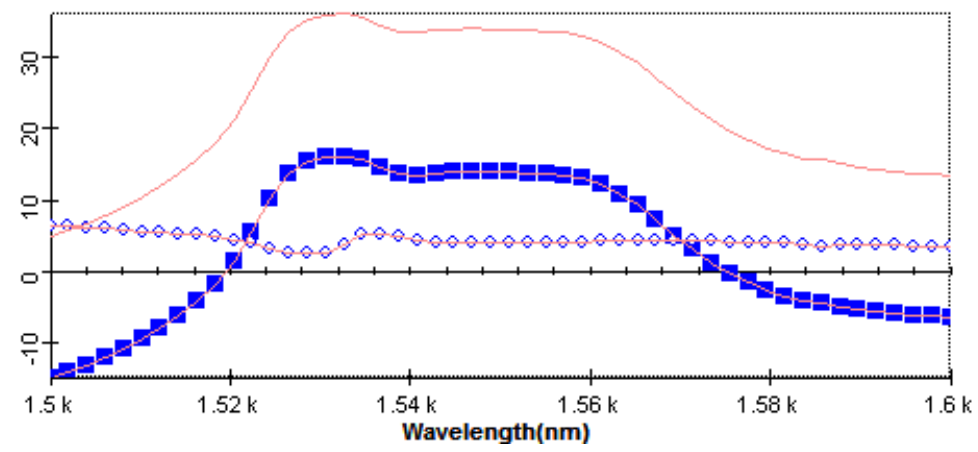

Figure 6. Gain curve / $\square$ Signal Power curve / $\odot$ Noise Figure curve

From the teaching effect of EDFA gain characteristics simulation, we can see there are many advantages of displaying the optoelectronic devices and fiber communication system to students by OptiSystem platform, for example, it can visualize the abstract concept, intuitive the complicated theory, help students understand the experimental principle and method, fully mobilize the enthusiasm of learning interest, steady professional knowledge, exercise and improve the practical ability and so on. 


\section{CONCLUSIONS}

The experimental teaching method based on OptiSystem simulation platform under the situation of lacking practical device, taking full use of the modern multi-media to teach in the class, combining the class with the computer operation, so that students take active part in class, mobilize the learning enthusiasm through the mode of experiment, discussing, peer reviewing. Comparing to the traditional teaching mode, this method not only emphasizes the teaching of knowledge and theory, but also can exercise the ability of team work and cultivates the spirit of innovation as well, which achieving the aim of students independent thinking and learning, largely improve the teaching effect. This research was supported by the Chongqing high school key teaching reform research project (Grant No.0824119).

\section{REFERENCES}

[1] Optiwave corporation, "OptiSystem Overview", https://optiwave.com/resources/applications-resources/opticalcommunication-system-design/

[2] Wang Qiuguang, Zhang Yalin, Hu Caiyun, Zhao Yingqi, “Application of OptiSystem simulation in experiment teaching of optical fiber communication", Laboratory Science 18(1), 26-29 (2015)

[3] Zhou Xuefang, Wang Tianshu, "Application of Simulation Software on the Course Fiber Optical Communications Experiment Teaching", Experiment Science and Technology 9(5), 53-56 (2011)

[4] Cui Xuerong, Cao Aiqing, Li Juan, Wu Chunle, "Application of research-oriented teaching mode in reform of experimental teaching methods", Experimental Technology and Management 33(1), 176-178 (2016)

[5] Li Hesheng, Jiang Xiamin, "Discussion and Practice of Teaching Methods for the Sake of Undergraduate Ability", Higher Education Forum 8, 63-65 (2010)

[6] Liu Zaiying, Yang Ping, Zhang Lixiao, "Probing into the practice teaching of programming design courses", Research And Exploration In Laboratory 32(10), 156-159 (2013)

[7] Jiang Tengjiao, "Simulation of EDFA gain factor by using Optisystem", Optical Communication Technology 8, $18-20(2013)$ 